\title{
Article
}

\section{Photoacoustic Imaging for Periodontal Disease Examination}

\author{
Mitrayana ${ }^{1, *}$, Atika Windra Sari ${ }^{1}$ and Rini Widyaningrum ${ }^{2}$ \\ 1 Department of Physics, Universitas Gadjah Mada, Yogyakarta 55281, Indonesia; atikawin- \\ drasari1@gmail.com \\ 2 Department of Dentomaxillofacial Radiology, Faculty of Dentistry, Universitas Gadjah Mada, Yogyakarta \\ 55281, Indonesia; rinihapsara@ugm.ac.id \\ * Correspondence: mitrayana@ugm.ac.id; Tel.: +62-156-854-834
}

\begin{abstract}
Inflammation of the periodontal tissue (periodontitis) is the highest problem of oral health in Indonesia after caries. Photoacoustic imaging (PAI) is a new imaging technique that can be simply constructed using a diode laser combined with a condenser microphone. This study aims to determine that a simple PAI system was able to image periodontal disease in animal model. Samples of the study were normal periodontal and periodontitis tissue, obtained from Sprague-Dawley rats that were divided into four groups, i.e. the control group, treatment group 1 (7 days periodontitis induction), treatment group 2 (11 days periodontitis induction), and treatment group 3 (14 days periodontitis induction). The PAI system was controlled by Labview and Arduino IDE software from a personal computer. Results of the study reveal that the optimal frequency of laser modulation for periodontal tissue imaging was $19 \mathrm{kHz}$ with duty cycle of $50 \%$. Photoacoustic (PA) intensity was obtained from higher to lower of $-68,71 \mathrm{~dB}$ (treatment group 3), $-70,69 \mathrm{~dB}$ (treatment group 2), $-71,69 \mathrm{~dB}$ (treatment group 1), and $-73,07 \mathrm{~dB}$ (control group) respectively. The photoacoustic images were analyzed to define the contrast between sample and media. The PA intensity of the samples were higher than media. Therefore, this study demonstrate the feasibility of simple PAI system to differentiate normal periodontal tissue and periodontitis.
\end{abstract}

Keywords: diode laser, imaging, periodontal, photoacoustic.

\section{Introduction}

Periodontal disease, also known as periodontitis, is a chronic inflammation disease that affects supporting tissue of the teeth [1,3]. Periodontal disease commonly caused by accumulation of pathogenic bacteria infection that induce the inflammation in gums (generally known as gingivitis) that subsequently leading in progressive destruction of periodontal ligament and alveolar bone [2,4]. If not treated properly, periodontal disease can caused systemic disease including coronary heart disease, stroke, and peripheral arterial disease [3].

The diagnosis of periodontal disease is based on clinical and X-rays radiographic examination. The $\mathrm{X}$-rays radiography has being excellent imaging modality for hard tissue examinations, but it has limitation for imaging the soft tissues. The photoacoustic imaging (PAI) is a new imaging technique that developed based on the photoacoustic effect, a phenomenon of acoustic signal generation from material being exposed by laser or other electromagnetic waves [4]. Photoacoustic imaging has been proven to be an effective technique for soft tissue imaging. Therefore, this imaging technique is potential to be developed as an imaging techniques for examination of periodontal disease $[5,6]$.

In last two decades, various non-invasive PAI systems have been developed in the field of biomedical research $[6,7,8,9,10]$. Photoacoustic imaging is a future biomedical imaging modality that combine the merits of optical resolution with the depth of acoustic penetration. This imaging technique has capacity to image structural, functional, molecular and kinetic information from various tissue. Photoacoustic imaging also has been developed for clinical applications. The rapid development of clinical applications of photo- 
acoustic imaging include breast cancer imaging, dermatological imaging, vascular imaging, carotid artery imaging, musculoskeletal imaging, gastrointestinal imaging, adipose and other tissue imaging [8,11].

Photoacoustic imaging (PAI) is a non-invasive and low-cost imaging technique. It does not produce ionizing radiation [12]. The electromagnetic source in PAI system is generally uses laser. The photoacoustic effect arises when the optical energy of laser interacts with medium. The energy is absorbed and converted into heat in the medium being exposed by the laser. Thermal expansion in the medium subsequently produce a pressure wave that will spread in the tissue. The pressure waves then propagates as acoustic waves that can be detected on the tissue and then reconstructed as photoacoustic image in the computer [13]. The development and application of lasers are widely used in the fields in medical and dentistry [5]. Previous study reveals that diode laser illumination is well absorbed by the gingival melanin pigment in the periodontal tissue [14].

The present study was carried out to demonstrate the capability of simple PAI system based on diode laser and condenser microphone to image the periodontal disease. The photoacoustic images obtained in this study were compared to differentiate the photoacoustic images of normal (healthy) periodontal tissue with the images of periodontitis condition in samples taken from animal models.

\section{Materials and Methods}

The protocols of this study was approved by the research ethics committee of the Faculty of Dentistry, Universitas Gadjah Mada (Ref. No. 00491/KKEP/FKGUGM/EC/2020). Samples in this study were periodontal tissue of male Sprague-Dawley (SD) rats, aged 4-8 weeks and weighing 150-200 grams. Rats were divided randomly into four groups according to Table 1. Each group consist of 5 rats.

Table 1. Groups of animal model for periodontal disease in the study

\begin{tabular}{ccc}
\hline Group & N & Treatment \\
\hline Control Group (C) & 5 & No treatment \\
Group (T1) & 5 & 7 days \\
Group (T2) & 5 & 11 days \\
Group (T3) & 5 & 14 days \\
\hline
\end{tabular}

The animals were acclimatized for seven days and kept in a room which had a constant temperature of $25^{\circ} \mathrm{C}$ with a 12-hour-light and 12-hour-darkness cycle before being treated. There was no treatment given for the control group. Silk ligatures were tied around the incisors of treatment groups. Before treatment, all animals in treatment groups were anesthetized with intramuscular injection of ketamine $(10 \mathrm{mg} / \mathrm{kgW})$ [15]. The method of periodontitis induction in the animal model of the study is presented in Figure 1. 


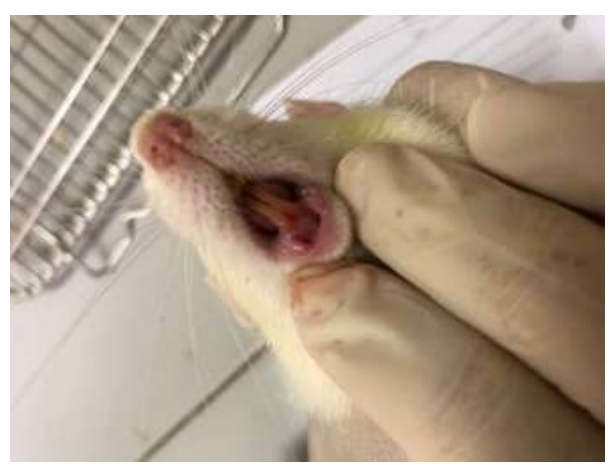

(a)

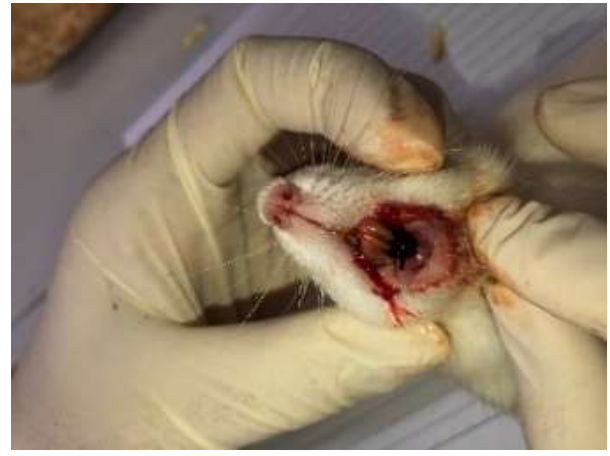

(b)

Figure 1 Teeth and gingiva condition in control group (a), silk ligatures tied around the incisors of treatment group (b).

After treatment, all rats were euthanized. The region of incisor and the supporting structures were dissected and fixed in $10 \%$ buffered formalin for 24 hours. The specimens were decalcified using EDTA $10 \%\left(\mathrm{pH} 7.4\right.$ ) for 1 week at $4^{\circ} \mathrm{C}$. The tissue specimens were then embedded in paraffin and cut in the median line.

The photoacoustic (PAI) system in this study is presented in Figure 2. The excitation source in the PAI system was a $532 \mathrm{~nm}$ diode laser with $200 \mathrm{~mW}$ peak power. The diode laser was modulated using the Arduino. The laser was illuminated onto the sample to generate the photoacoustic signal. Photoacoustic signal was then detected by a condenser microphone ECM8000 (Behringer, Germany) and was amplified using a soundcard connected with a personal computer. The PAI system is described more specific in the previous study [16].

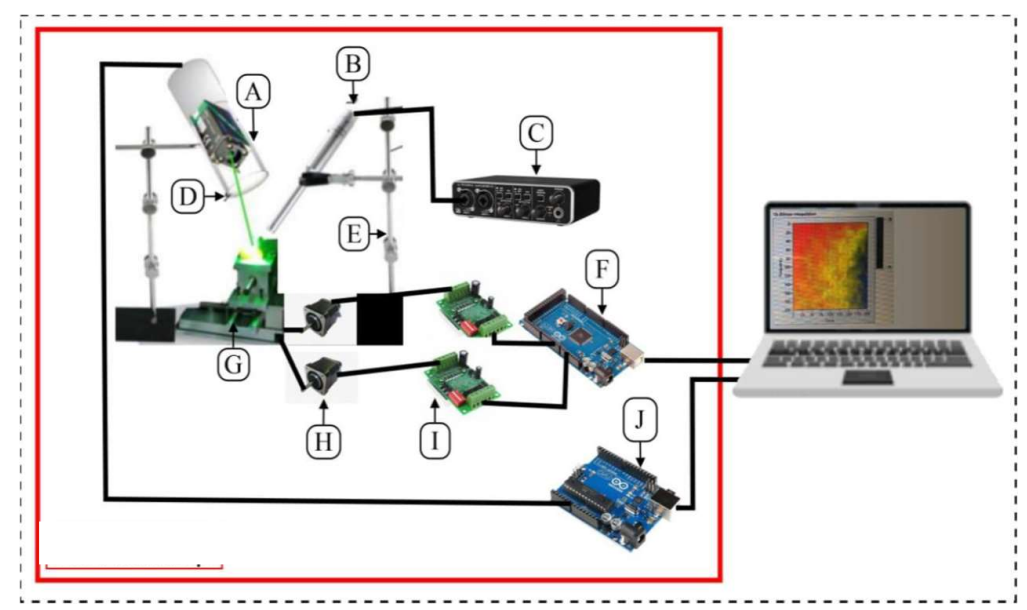

Figure 2 Schematic depiction of experimental setup for photoacoustic imaging. A. Diode laser, B. Condenser microphone, C. Soundcard, D. Lens, E. Stative, F. Arduino mega, G. Sample, H. Motor stepper, I. Motor driver, J. Arduino uno [17].

To ensure that the PAI system can be used for periodontal tissue imaging, the characterization of laser power stability was performed to illuminate the sample for 15 minutes. Analysis of laser power stability can be observed in Figure 4 that display the laser output power during sample scanning. Meanwhile the calibration of condenser microphone also conducted to ensure that the frequency given by the acoustic source was in the same frequency detected by the microphone that recorded in Labview. Figure 5 confirmed the relationship between the frequency of the sound source $\left(f_{g}\right)$ with the frequency received by the microphone $\left(f_{m}\right)$. 
Characterization of $x-y$ stage is principal to determine the accuracy of the stage shifts towards $x$ and $y$ direction following to the number of steps inputted in the Labview program. The procedures to characterize the $x-y$ stage shifts was performed by giving input of 10,20,30, and 40 steps in the Labview program, then the length of the $x-$ $y$ stage shifts were measured using digital caliper.

The flowchart of the study is presented in Figure 3. To determine the optimal laser modulation for periodontal tissue imaging in this study, laser was exposed onto the sample with various frequency and duty cycle. Variations of frequency to determine the optimal laser modulation were $17 \mathrm{kHz}, 18 \mathrm{kHz}, 19 \mathrm{kHz}$, and $20 \mathrm{kHz}$. Whereas variations of duty cycle were $10 \%, 20 \%, 30 \%, 40 \%$, and $50 \%$, respectively.

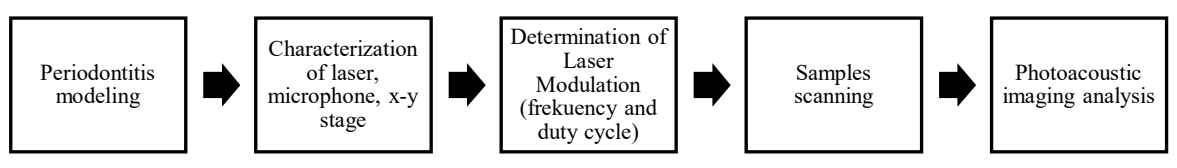

Figure 3 The flowchart of the study.

\section{Results and Discussion}

The animal model in this study was Sprague-Dawley rats. The periodontal tissue of Sprague Dawley rats can be induced using a simple method, so that is can be used in periodontitis modeling that mimic the periodontitis condition in humans [18].

The PAI system is generally built by integrating ultrasound transducer as detector with a pulsed laser as an electromagnetic wave source [9]. Acoustic signals in this study are generated by using a modulated continuous-wave (CW) laser as an electromagnetic wave source [16]. Modulated excitation on CW lasers was carried out by regulating the duty cycle and pulse width periodically at a single frequency using pulse width modulation (PWM) technique. This technique was applied to obtain fluctuating laser radiation $[11,16]$ in this study.

When a laser is modulated with a certain duty cycle, the output pulse was emitted in a sporadic manner (non-stationary pulses). Non-stationary pulses cause thermal expansion in the sample, which resulted in an acoustic signal. The frequency of resulting acoustic signals in our experiment were in the same frequency of laser modulation, and it was also directly proportional to the energy absorption. The duty cycle of laser modulation was proportional to the intensity of the thermal expansion that generate the acoustic signals [13].

Figure 4 displays the relationship between laser output power and exposure time. After 15 minutes of scanning, it was discovered that the laser power output had barely changed. The laser power almost does not changing during scanning time so that the laser exposure was assumed in stable condition during photoacoustic imaging in this study. 


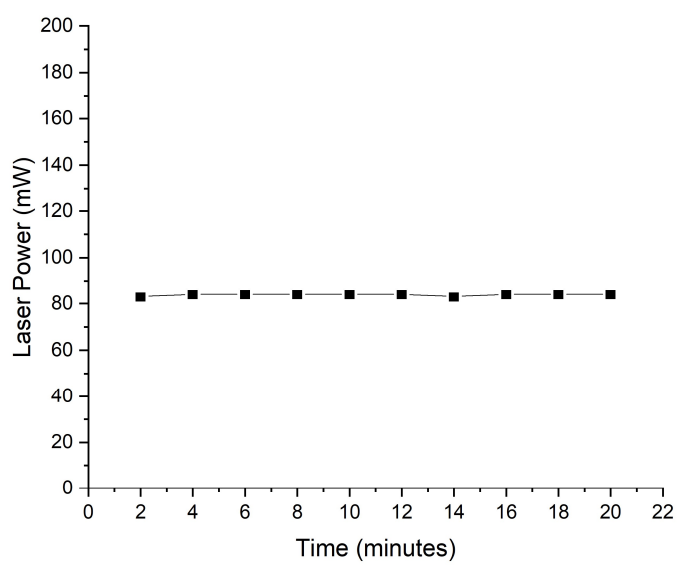

Figure 4 Characterization of main component in the PAI system: Laser power $(\mathrm{mW})$ observed in respective interval time.

The characterization of microphone in Figure 5 reveals a linear relationship between frequency received by microphone $\left(f_{m}\right)$ and frequency emitted by sound generator $\left(f_{g}\right)$ (Figure 5) and the frequency received by the microphone was represented by the $f_{m}$ data. The frequency produced from the sound generator was represented by $f_{g}$ data. The signal detected by the microphone was in the form of a voltage, which was read by the Labview program. The signal was converted from a function of time into a function of frequency using the Fast Fourier Transform (FFT) in the Labview program.

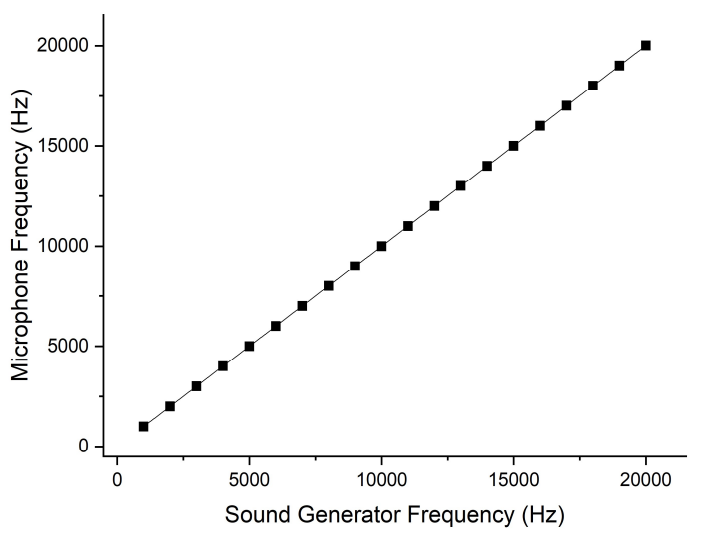

Figure 5 Characterization of main component in the PAI system: correlation of sound generator frequency $\left(f_{g}\right)$ with microphone frequency $\left(f_{m}\right)$.

The correlation between input step in Labview program with the step number is in a linear relationship (Figure 6). The result of the experiment in Figure 6 indicates that the input in Labview program was precisely corresponds to the shift of $x-y$ stage. 


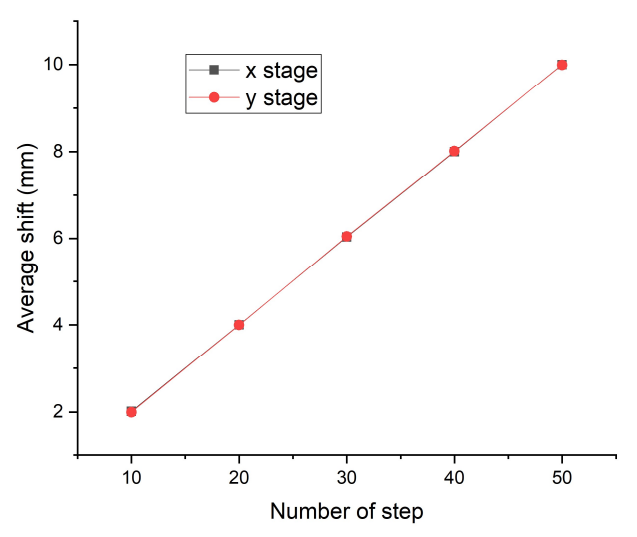

Figure 6 Characterization of main component in the PAI system: the average shift of $x-y$ stage step for each $0.2 \mathrm{~mm}$ input in Labview.

The laser in the PAI system was modulated to induce the acoustic signals from the sample in this study. The energy absorbed by the sample was comparable to the photoacoustic signal. The duty cycle of laser modulation was a percentage of the signal's active time during the laser modulation. The best periodontal images were obtained when the laser was modulated with optimum frequency and duty cycle that capable to generate the higher PA intensity from the samples. Figure 7 shows that the best frequency for scanning the sample of periodontal tissue was $19 \mathrm{kHz}$ (Figure 7a), with a duty cycle of 50\% (Figure $7 b)$.

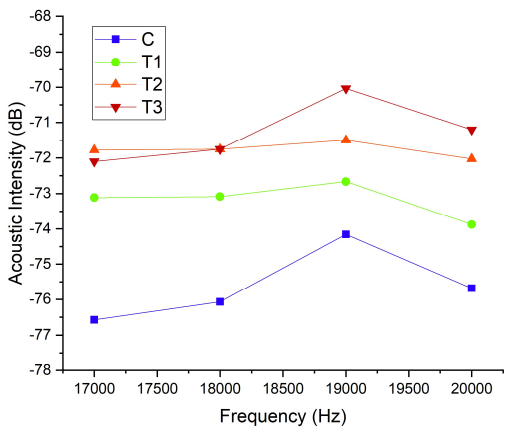

(a)

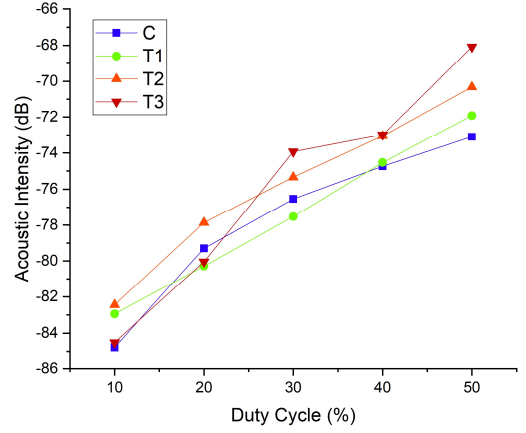

(b)

Figure 7 The PA intensity generates from periodontal tissue been illuminated in respective frequency (a) and duty cycle (b) of laser modulation.

The aim of this study is to differentiate healthy periodontal tissue and periodontitis tissue in terms of photoacoustic image and average PA intensity. The PAI system was able to scan samples at a rate of one second per pixel. Data of acoustic signals were collected from samples with a resolution of $30 \times 30$ pixels on average. It took about 30 minutes to scan every sample. Data of the PA intensity were used to create photoacoustic image in this study. Image color was used to describe the PA intensity produced from the PAI system in this study. Result of this study is in accordance with previous study that low PA intensity was displayed as blue, medium PA intensity was yellow, and high PA intensity was red $[15,16]$. The difference in PA intensity between the control group and treatment group were shown in figure 8 .

Figure 8 displays the average PA intensity of the sample, which reveals that the average of PA intensity of periodontal tissue in the treatment group was greater than healthy 
periodontal tissue in the control group. The groups of $\mathrm{C}, \mathrm{T} 1, \mathrm{~T} 2$, and $\mathrm{T} 3$ have average PA intensity values of $-73.07 \mathrm{~dB},-71.69 \mathrm{~dB},-70.34 \mathrm{~dB}$, and $-68.71 \mathrm{~dB}$, respectively. The inflammation of periodontal tissue is causing alteration of mineral distribution, which revealed a greater PA signal among the treatment groups than the control group in the supporting tissue of the teeth [7].

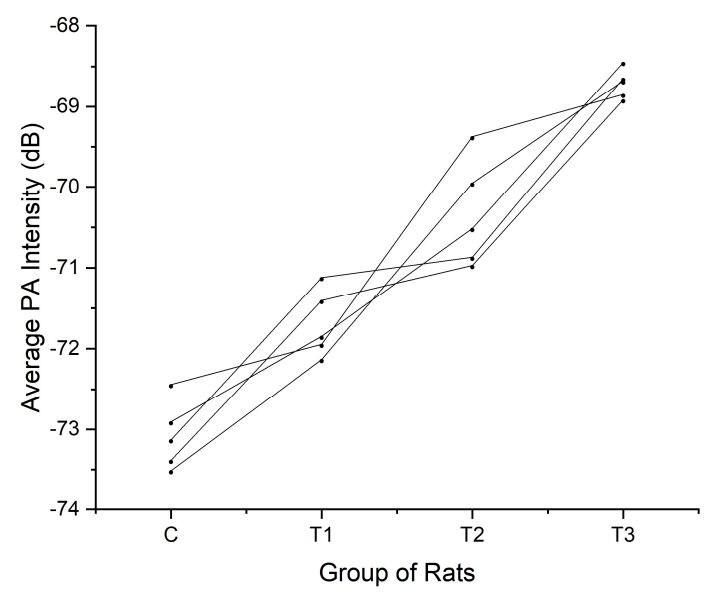

Figure 8 The PA intensity of photoacoustic images from control and treatment groups.

Figures 8 illustrates the difference in PA intensity between control and treatment groups. The photoacoustic of periodontal tissue samples in this study were shown in Figure 9. The photoacoustic images in Figure 9 were indicate different color depending on the PA intensity. Blue denotes a low level of PA intensity, yellow a moderate level of PA intensity, and red a high level of PA intensity from samples. It was recognized from Figure 9 that the control group display lower PA intensity based on the PA intensity (Figure 8) and the color of the photoacoustic image (Figure 9). Yellow dominates the photoacoustic images of control group. Whereas the photoacoustic images of periodontal disease (periodontitis) tissue in treatment groups display a high PA intensity, which showed up in the picture as a red hue.
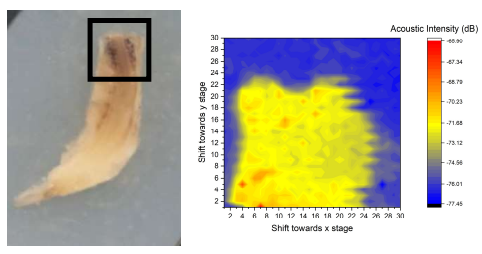

(a)
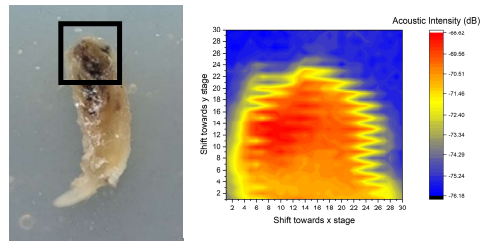

(c)
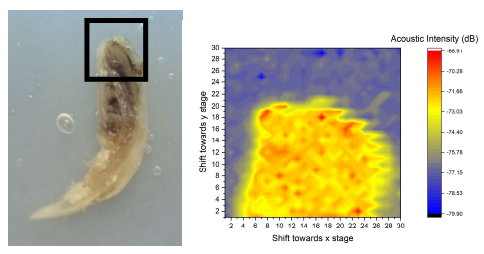

(b)
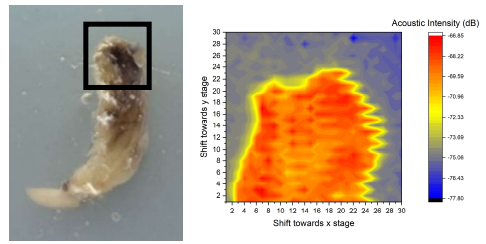

(d)

Figure 9 Incisors specimens of Sprague Dawley rat's periodontal tissue and photoacoustic images: (a) Control group (C), (b) Group 1 (T1), (c) Group 2 (T2), (d) Group 3 (T3). The black square in photographs shows the area been scanned for photoacoustic imaging in this study. 


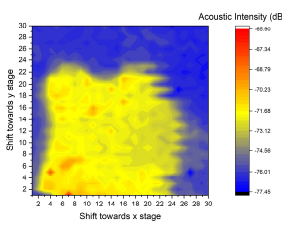

(a)

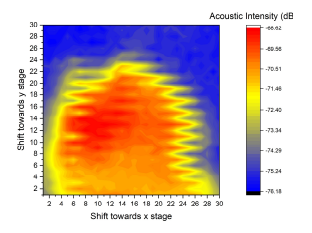

(c)

Periodontitis is chronic disease that caused by bacterial infection. Periodontitis induction by using silk ligatures [19] can cause accumulation of bacteria then induce of tooth supporting inflammation and destruction in periodontal tissue followed by demineralization [5].

The PA intensity of the periodontitis (treatment) group was higher than the PA intensity of the control group, as shown in Figure 8. This was may be caused by different mineral distributions in the tissue that caused by inflammation [6]. The laser energy was more easily absorbed by inflamed tissue in treatment groups, resulting in a greater average PA intensity [7].

The density of certain tissues or materials also affects the laser absorption in photoacoustic imaging. Chromophores are tissue components that can absorb laser light energy [20]. Several chromophores, such as hydroxyapatite, protein, and blood vessels can be found in oral tissue [21]. The periodontal tissue has a lower tissue mass than the teeth. As a result, periodontal tissue has a higher optical absorption than teeth. Periodontal structure was consist of hard and soft tissues. There are several blood vessels and nerves in soft tissue [22]. Hemoglobin is a powerful chromophore found in blood, and it may found more in periodontitis condition than healthy periodontal tissue.

The contrast of photoacoustic image was formed by PA intensity differences. This can be used as an initial parameter, indicating that the photoacoustic imaging system was able to distinguish between healthy periodontal and periodontitis condition.

Figure 10 shows different PA intensity between periodontal tissue and media. The graphs in Figure 10 shows that PA intensity of periodontal tissue from $5^{\text {th }}$ until $25^{\text {th }}$ steps of $x$ direction at the $10^{\text {th }}$ step of $y$ direction are tend to be higher than PA intensity of media. Therefore, the PA images can represent different PA intensity between periodontal tissue and media.
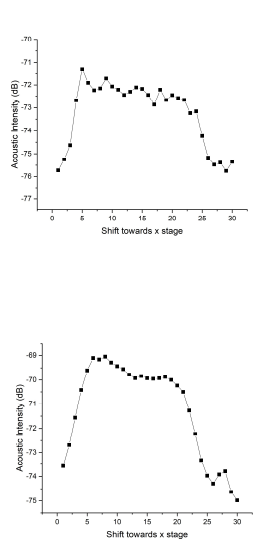

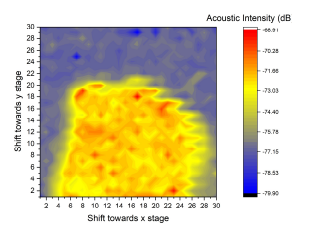

(b)

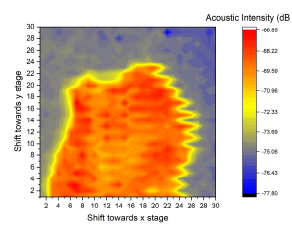

(d)
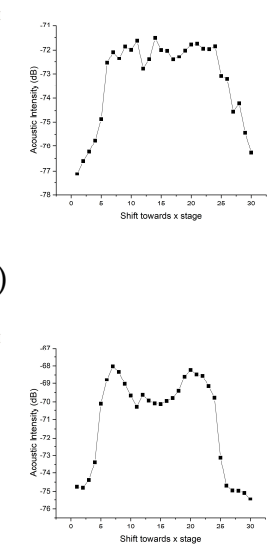

Figure 10 Different PA intensity between periodontal tissue and medium. (a) Control group (C), (b) Group 1 (T1), (c) Group 2 (T2), (d) Group 3 (T3).

\section{Conclusions}

A photoacoustic imaging system based on diode laser and condenser microphone in this study is capable for imaging the periodontal disease. Based on the results of this study, the PA intensity of the photoacoustic images from treatment groups was higher than control group. The optimal frequency of laser modulation for imaging the periodontal tissue was $19 \mathrm{kHz}$ with duty cycle of $50 \%$. The simple photoacoustic system in this study can be developed further to build photoacoustic tomography system.

Author Contributions: Conceptualization, M.; Data curation, A.W.S.; Formal analysis, M. and R.W.; Funding acquisition, M.; Investigation, A.W.S.; Methodology, M; Project administration, M.; Resources, M.; Supervision, M. and R.W.; Validation, R.W.; Visualization, M. and A.W.S.; Writing- 
original draft, M.; Writing - review and editing, R.W. All authors have read and agreed to the published version of the manuscript.

Funding: This research was funded by Lembaga Pengelola Dana Pendidikan and Penelitian Terapan Unggulan Perguruan Tinggi Programs of the Ministry of Education of the Republic of Indonesia.

Acknowledgments: We would like to thank Iman R. Santoso and Mirza Satriawan for his valuable suggestion and discussion.

Conflicts of Interest: The authors declare no conflict of interest.

\section{References}

1. J. S. Borges et al., "Does systemic oral administration of curcumin effectively reduce alveolar bone loss associated with periodontal disease? A systematic review and meta-analysis of preclinical in vivo studies," J. Funct. Foods, vol. 75, no. October, p. 104226, 2020, doi: 10.1016/j.jff.2020.104226.

2. R. Aquino-martinez et al., "LPS-induced premature osteocyte senescence : Implications in in fl ammatory alveolar bone loss and periodontal disease pathogenesis," Bone, vol. 132, no. December 2019, p. 115220, 2020, doi: 10.1016/j.bone.2019.115220.

3. G. Seinost et al., "Periodontal treatment and vascular inflammation in patients with advanced peripheral arterial disease : A randomized controlled trial," Atherosclerosis, vol. 313, no. September, pp. 60-69, 2020, doi: 10.1016/j.atherosclerosis.2020.09.019.

4. Q. Yao, Y. Ding, G. Liu, and L. Zeng, “Low-cost photoacoustic imaging systems based on laser diode and light-emitting diode excitation," J. Innov. Opt. Health Sci., vol. 10, no. 4, pp. 1-13, 2017, doi: 10.1142/S1793545817300038.

5. Newman, Takei, Klokkevold, and Carranza, Clinical Periodontology. Philadelphia: Elseiver, 2019.

6. M. Erfanzadeh, P. D. Kumavor, and Q. Zhu, "Laser scanning laser diode photoacoustic microscopy system," Photoacoustics, vol. 9, pp. 1-9, 2018, doi: 10.1016/j.pacs.2017.10.001.

7. V. Periyasamy, M. Rangaraj, and M. Pramanik, "Photoacoustic imaging of teeth for dentine imaging and enamel characterization," vol. 10473, p. 8, 2018, doi: 10.1117/12.2286733.

8. A. B. E. Attia et al., "A review of clinical photoacoustic imaging: Current and future trends," Photoacoustics, vol. 16, no. August, p. 100144, 2019, doi: 10.1016/j.pacs.2019.100144.

9. T. Zhao, A. E. Desjardins, S. Ourselin, T. Vercauteren, and W. Xia, “Minimally invasive photoacoustic imaging: Current status and future perspectives," Photoacoustics, vol. 16, no. June, p. 100146, 2019, doi: 10.1016/j.pacs.2019.100146.

10. C. Moore et al., "Photoacoustic imaging for monitoring periodontal health: A first human study," Photoacoustics, vol. 12, no. November, pp. 67-74, 2018, doi: 10.1016/j.pacs.2018.10.005.

11. C. Y. Lin, F. Chen, A. Hariri, C. J. Chen, T. Takesh, and J. V Jokerst, "Photoacoustic Imaging for Noninvasive Periodontal Probing Depth Measurements," 2018, doi: 10.1177/0022034517729820.

12. Y. Wu, H. K. Zhang, J. Kang, and E. M. Boctor, "An economic photoacoustic imaging platform using automatic laser synchronization and inverse beamforming," Ultrasonics, vol. 103, no. October 2019, p. 106098, 2020, doi: 10.1016/j.ultras.2020.106098.

13. L. V Wang, Photoacoustic Imaging and Spectroscopy. Boca Raton: CRC Press, 2017.

14. M. Taher Agha and P. Polenik, "Laser Treatment for Melanin Gingival Pigmentations: A Comparison Study for 3 Laser Wavelengths 2780, 940, and 445 nm," Int. J. Dent., vol. 2020, 2020, doi: 10.1155/2020/3896386.

15. E. Nelissen et al., "Validation of the xylazine/ketamine anesthesia test as a predictor of the emetic potential of pharmacological compounds in rats," Neurosci. Lett., vol. 699, no. June 2018, pp. 41-46, 2019, doi: 10.1016/j.neulet.2019.01.026.

16. R. Widyaningrum, D. Agustina, M. Mudjosemedi, and Mitrayana, "Photoacoustic for Oral Soft Tissue Imaging based on Intensity Modulated Continuous-Wave Diode Laser," vol. 8, no. 2, pp. 622-627, 2018.

17. A. W. Sari, "Application of Photoacoustic Imaging for Periodontal Disease Examination (ex-vivo study in Sprague-Dawley Rats)," Universitas Gadjah Mada, 2021.

18. K. Jeong-hyon, G. Bon-hyuk, N. Sang-soo, and P. Yeon-cheol, "Journal of Traditional Chinese Medical Sciences A review of rat models of periodontitis treated with natural extracts," J. Tradit. Chinese Med. Sci., no. xxxx, pp. 1-9, 2020, doi: 10.1016/j.jtcms.2020.05.005.

19. Chiang et al., "A Salvia miltiorrhiza ethanol extract ameliorates tissue destruction caused by experimental periodontitis in rats," no. 1, pp. 133-139, 2016, doi: 10.1111/jre.12292.

20. M. Kim, G. Jeng, M. O. Donnell, and I. Pelivanov, "Photoacoustics Correction of wavelength-dependent laser fluence in sweptbeam spectroscopic photoacoustic imaging with a hand-held probe," Photoacoustics, vol. 19, no. May, p. 100192, 2020, doi: 10.1016/j.pacs.2020.100192.

21. W. Rui, C. Tao, and X. Liu, “Ultrasonics - Sonochemistry Multiple information extracted from photoacoustic radio-frequency signal and the application on tissue classification," Ultrason. - Sonochemistry, vol. 66, no. March, p. 105095, 2020, doi: 10.1016/j.ultsonch.2020.105095.

22. W. L. Chai, M. Razali, K. Moharamzadeh, and M. S. Zafar, The hard and soft tissue interfaces with dental implants. Elsevier Ltd, 2020 . 